\title{
Number of fermion generations from a novel grand unified model
}

\author{
Pritibhajan Byakti $^{1}$, David Emmanuel-Costa ${ }^{2, a}$, Arindam Mazumdar ${ }^{1}$ Palash B. Pal $^{1}$ \\ ${ }^{1}$ Saha Institute of Nuclear Physics, Kolkata 700064, India \\ ${ }^{2}$ Departamento de Física and Centro de Física Teórica de Partículas (CFTP), Instituto Superior Técnico (IST), Universidade de Lisboa, \\ Av. Rovisco Pais, 1049-001 Lisbon, Portugal
}

Received: 31 October 2013 / Accepted: 23 December 2013 / Published online: 31 January 2014

(C) The Author(s) 2014. This article is published with open access at Springerlink.com

\begin{abstract}
Electroweak interactions based on the gauge group $\mathrm{SU}(3)_{\mathrm{L}} \times \mathrm{U}(1)_{\mathrm{X}}$, coupled to the $\mathrm{QCD}$ gauge group $\mathrm{SU}(3)_{\mathrm{c}}$, can predict the number of generations to be multiples of three. We first try to unify these models within $\mathrm{SU}(N)$ groups, using antisymmetric tensor representations only. After examining why these attempts fail, we continue to search for an $\mathrm{SU}(N)$ GUT that can explain the number of fermion generations. We show that such a model can be found for $N=9$, with fermions in antisymmetric rank- 1 and rank-3 representations only, and we examine the constraints on various masses in the model coming from the requirement of unification.
\end{abstract}

\section{Introduction}

In the standard model, the number of fermion generations appears as an arbitrary parameter, meaning that a mathematically consistent theory can be built up using any number of fermion generations. The same is true for many extensions of the standard model, including grand unification models based on the gauge groups $\mathrm{SU}(5)$ and $\mathrm{SO}(10)$. An interesting question related to the extension of the standard model is whether the number of fermion generations can in any way be explained through the internal consistency of the model. In the literature, there is some discussion of grand unification models based on large orthogonal groups like $\mathrm{SO}(18)$, where one spinor multiplet contains all known fermion fields of all generations, and much more [1]. It was shown that [2,3], with a suitable symmetry breaking scheme, only three generations can remain light, whereas the others obtain masses at much above the electroweak scale.

In a quite different line of development, it was shown that if one extends the electroweak group to $\mathrm{SU}(3) \times \mathrm{U}(1)$ and tries to accommodate the standard fermions into multiplets of this gauge group which must include some new fermions,

a e-mail: david.costa@tecnico.ulisboa.pt cancelation of gauge anomalies can restrict the number of generations and one obtains consistent models with the number of generations equal to three or any multiple of it [4-8].

These models are described briefly in Sect. 2. Then, in Sects. 3 and 4, we try to see whether these models can be embedded into a simple SU(N) group. We conclude that if one uses only completely antisymmetric tensor representations, such an embedding cannot be found. Then, in Sect. 5, we start looking for general conditions that will specify the number of fermion generations for arbitrary $\mathrm{SU}(\mathrm{N})$ groups. In Sect. 6, we analyze one simple model, based on the group SU(9), which gives three generations. The renormalization group analysis of various scales in these models is performed in Sect. 7. We end with some concluding remarks in Sect. 8.

\section{The 3-3-1 models}

The 3-3-1 models are based on the gauge group $\mathrm{SU}(3)_{\mathrm{c}} \times$ $\mathrm{SU}(3)_{\mathrm{L}} \times \mathrm{U}(1)_{\mathrm{X}}$. The first factor in the gauge group is the group of QCD, whereas the other two factors pertain to electroweak interactions. There are two versions of such models, and we discuss them one by one.

In one version, proposed by Pisano and Pleitez [5] and by Frampton [6] (to be denoted as the PPF model), the left-chiral fermions and antifermions belong to the following representations of the gauge group:

$$
\begin{aligned}
f_{a} & =\left(\begin{array}{l}
\hat{\ell} \\
v_{\ell} \\
\ell
\end{array}\right)_{a} \sim[1,3,0] \\
Q_{1} & =\left(\begin{array}{l}
T_{1} \\
u_{1} \\
d_{1}
\end{array}\right) \sim\left[3,3, \frac{2}{3}\right] \\
Q_{i} & =\left(\begin{array}{l}
B \\
d \\
u
\end{array}\right)_{i} \sim\left[3,3^{*},-\frac{1}{3}\right]
\end{aligned}
$$


$\hat{u}_{a} \sim\left[3^{*}, 1,-\frac{2}{3}\right]_{a}$

$\hat{d}_{a} \sim\left[3^{*}, 1, \frac{1}{3}\right]_{a}$

$\hat{T}_{1} \sim\left[3^{*}, 1,-\frac{5}{3}\right]$

$\hat{B}_{i} \sim\left[3^{*}, 1, \frac{4}{3}\right]_{i}$.

Note that there are two kinds of generation indices: $a$ goes from 1 to 3, whereas $i$ takes only the values 2 and 3. An antifermion has been denoted by a hat. We emphasize that all representations given above pertain to the left-chiral components only. The representation of a right-chiral fermion would be the complex conjugate of that of the left-chiral antifermion, and vice versa. Note that there are extra quark fields, i.e., fields which are triplets of $\mathrm{SU}(3)_{\mathrm{c}}$, but there is no neutrino field that is sterile under the standard model. Different generations of fermions are not copies of one another. Gauge anomalies cancel between the three generations $[5,6]$, but not within a single generation. Thus, the consistency of the model requires three generations of fermions. Of course, this pattern of three generations can be repeated, so one would obtain a number of generations that is a multiple of three.

The other version of 3-3-1 models was proposed by Singer et al. [4] (and hence will be referred to as the SVS model) and was examined by other authors later $[7,8]$. In this version, there are sterile neutrinos, the left-chiral component of which has been denoted by $\hat{v}$ in the list below:

$$
\begin{aligned}
& f_{a}=\left(\begin{array}{l}
v_{\ell} \\
\ell \\
\widehat{v}_{\ell}
\end{array}\right)_{a} \sim\left[1,3,-\frac{1}{3}\right] \\
& \hat{\ell}_{a} \sim[1,1,1] \\
& Q_{1}=\left(\begin{array}{l}
u_{1} \\
d_{1} \\
u_{1}^{\prime}
\end{array}\right) \sim\left[3,3, \frac{1}{3}\right] \\
& Q_{i}=\left(\begin{array}{l}
d \\
u \\
d^{\prime}
\end{array}\right)_{i} \sim\left[3,3^{*}, 0\right] \\
& \hat{u}_{a}, \hat{u}_{1}^{\prime} \sim\left[3^{*}, 1,-\frac{2}{3}\right] \\
& \hat{d}_{a}, \hat{d}_{i}^{\prime} \sim\left[3^{*}, 1, \frac{1}{3}\right] .
\end{aligned}
$$

The notation for the generation indices is as before. The primed fields are extra quark fields, which are not present in the standard model. Like the previous model, gauge anomalies cancel between various generations.

The difference between the two models may be summarized in the following way. The standard model gauge group is not a maximal subgroup of the group $\mathrm{SU}(3)_{\mathrm{c}} \times \mathrm{SU}(3)_{\mathrm{L}} \times$
$\mathrm{U}(1)_{\mathrm{X}}$. In particular, the electroweak $\mathrm{SU}(3)_{\mathrm{L}}$ has two neutral generators, and some combination of these two, along with the generator of $\mathrm{U}(1)_{\mathrm{X}}$, form $Y$ and $I_{3 L}$, the two neutral generators of the standard electroweak model. Using the standard normalization of the SU(3) generators,

$\operatorname{tr}\left(T_{A} T_{B}\right)=\frac{1}{2} \delta_{A B}$,

these combinations are given by

$I_{3 L}=-\frac{1}{2} T_{3}+\frac{\sqrt{ } 3}{2} T_{8}$,
$Y=\frac{3}{2} T_{3}+\frac{\sqrt{ } 3}{2} T_{8}+X$

in the first model, whereas in the second one, they are given by

$I_{3 L}=T_{3}$,

$Y=-\frac{1}{\sqrt{ } 3} T_{8}+X$.

The SU(3) generators are given by

$T_{i}= \begin{cases}\frac{1}{2} \lambda_{i} & \text { for the fundamental representation, } \\ -\frac{1}{2} \lambda_{i}^{*} & \text { for the anti-fundamental representation, }\end{cases}$

where the $\lambda$ are the well-known Gell-Mann matrices.

\section{Seeking embeddings into SU(6)}

Since the group $\mathrm{SU}(3)_{\mathrm{c}} \times \mathrm{SU}(3)_{\mathrm{L}} \times \mathrm{U}(1)_{\mathrm{X}}$ is of rank 5, the smallest unitary group that contains it as a subgroup is SU(6). Therefore, in this section, we analyze whether the models discussed in Sect. 2 can be embedded into an SU(6) grand unified model.

For the sake of convenience, let us announce here the notation that will be used for denoting representations of various groups. For the grand unified group, the notations will be denoted by boldface. The $\mathrm{SU}(3)_{\mathrm{c}} \times \mathrm{SU}(3)_{\mathrm{L}} \times \mathrm{U}(1)_{\mathrm{X}}$ representations will be denoted by three numbers in parentheses, e.g., $[a, b, c]$, as has already been done in Eqs. (1) and (2). The representation of the standard model group, when required, will be denoted by curly brackets, e.g., $\{a, b, c\}$. Thus, with this notation, the fundamental representation of SU(6) has the decomposition

$$
\begin{aligned}
\mathbf{6} & \rightarrow\left[3,1,-\frac{1}{3}\right]+\left[1,3, \frac{1}{3}\right] \\
& \rightarrow\left\{3,1,-\frac{1}{3}\right\}+\left\{1,2, \frac{1}{2}\right\}+\{1,1,0\} .
\end{aligned}
$$

It is now easy to see that neither the PPF nor the SVS model can be embedded into SU(6). For the PPF model [5,6], note the representation of leptons given in Eq. (1a). Certainly, it is not contained in the fundamental representation of SU(6) 
or its complex conjugate, $\mathbf{6}^{*}$. Higher representations can be obtained by taking Kronecker products of the $\mathbf{6}$ and $\mathbf{6}^{*}$ representations, and they will be of the generic form $(\mathbf{6})^{m}\left(6^{*}\right)^{n}$. Denoting the two different $\mathrm{SU}(3)_{\mathrm{c}} \times \mathrm{SU}(3)_{\mathrm{L}} \times \mathrm{U}(1)_{\mathrm{X}}$ representations that appear in Eq. (7) by $A$ and $B$, we can write

$(\mathbf{6})^{m}\left(\mathbf{6}^{*}\right)^{n} \rightarrow \sum_{m^{\prime}, n^{\prime}}\left(\begin{array}{c}m \\ m^{\prime}\end{array}\right)\left(\begin{array}{l}n \\ n^{\prime}\end{array}\right)(A)^{m-m^{\prime}}(B)^{m^{\prime}}\left(A^{*}\right)^{n-n^{\prime}}\left(B^{*}\right)^{n^{\prime}}$.

Take any term in the sum. Contributions to the $\mathrm{U}(1)_{\mathrm{X}}$ quantum number come from all four factors, and it is given by

$X=\frac{1}{3}\left(-m+2 m^{\prime}+n-2 n^{\prime}\right)$.

Thus, for the lepton representation such as in Eq. (1a), we need

$m-n=2\left(m^{\prime}-n^{\prime}\right)$.

in order to obtain $X=0$. Only the $B$ and $B^{*}$ contributes to non-trivial $\mathrm{SU}(3)_{\mathrm{L}}$ representations. In order to obtain a triplet, we need

$m^{\prime}-n^{\prime}=1 \bmod 3$

considering the triality of the representations. Moreover, the lepton must be a color singlet, which means that we should have

$\left(m-m^{\prime}\right)-\left(n-n^{\prime}\right)=0 \bmod 3$.

These three conditions cannot be satisfied with integers, and hence it is impossible to obtain a $(1,3,0)$ representation of $\mathrm{SU}(3)_{\mathrm{c}} \times \mathrm{SU}(3)_{\mathrm{L}} \times \mathrm{U}(1)_{\mathrm{X}}$ in any representation of $\mathrm{SU}(6)$.

For the SVS model, the same kind of analysis can be performed keeping an eye towards the antilepton in Eq. (2a). In order to produce a singlet of both SU(3) factors, one needs the Kronecker product of equal numbers of 6 and $6^{*}$. However, such products will give $X=0$.

We have thus proved that neither the PPF, nor the SVS model can be embedded into SU(6). This result should not be understood to imply that an SU(6) grand unified model is impossible. We can take a different embedding of the standard model generators $I_{3 L}$ and $Y$, viz.,

$I_{3 L}=T_{3}$,

$Y=\frac{1}{\sqrt{ } 3} \lambda_{8}+X$

The SM reduction of the $6^{*}$ representation of SU(6) can easily be read from Eq. (7):

$\mathbf{6}^{*} \rightarrow \underbrace{\left\{3^{*}, 1, \frac{1}{3}\right\}}_{\hat{d}}+\underbrace{\left\{1,2,-\frac{1}{2}\right\}}_{L}+\{1,1,0\}$.
Also, the antisymmetric rank-2 representation has the following decomposition under the SM gauge group:

$$
\begin{aligned}
\mathbf{1 5} \rightarrow & {\left[3^{*}, 1,-\frac{2}{3}\right]+\left[1,3^{*}, \frac{2}{3}\right]+[3,3,0] } \\
\rightarrow & \underbrace{\left\{3^{*}, 1,-\frac{2}{3}\right\}}_{\hat{u}}+\left\{1,2, \frac{1}{2}\right\}+\underbrace{\{1,1,1\}}_{\hat{\ell}}+\underbrace{\left\{3,2, \frac{1}{6}\right\}}_{Q} \\
& +\left\{3,1,-\frac{1}{3}\right\} .
\end{aligned}
$$

In both Eqs. (14) and (15), we have marked the known fermions which correspond to the SM representations. We observe that all known fermions of a single generation belong to these two representations. This is therefore like the minimal SU(5) grand unified model where the corresponding representations contain all known fermion fields of a single generation and nothing more. In this case, there are some extra fermions to complete the SU(6) representations.

There is one big difference between the SU(5) and this SU(6) model. This can be seen from the anomaly coefficients of different representations. For the completely antisymmetric tensorial representations of SU(N), the anomaly coefficients are as follows [9]:

\begin{tabular}{c|ccc}
\hline Representation & $\llbracket 1 \rrbracket$ & $\llbracket 2 \rrbracket$ & $\llbracket 3 \rrbracket$ \\
\hline anomaly coefficient & 1 & $N-4$ & $\frac{1}{2}(N-3)(N-6)$ \\
\hline
\end{tabular}

Here, the completely antisymmetric representation of rank $n$ has been denoted by $\llbracket n \rrbracket$. The anomaly coefficient of any representation and its complex conjugate will be the negatives of each other. We see that, for the SU(5) group, anomalies cancel between the $\mathbf{5}^{*}$ and $\mathbf{1 0}$ representations. For the SU(6) gauge group, the $\mathbf{6}^{*}$ and the $\mathbf{1 5}$ representations have anomaly coefficients of -1 and 2 , respectively. Thus, along with three copies of the $\mathbf{1 5}$ representation, which is necessary in order to have three quark doublets, we need six copies of the $6^{*}$ representation in order to cancel anomalies. There will thus be three extra copies of the lepton doublet $L$. But these will form gauge invariant masses with the three copies of the $\left\{1,2, \frac{1}{2}\right\}$ representation that appear in the three 15 -plets, and these masses can be much heavier than the electroweak scale. Similarly, among the six copies of the $\hat{d}$ representation that appear in the $6^{*}$ multiplets, three will form gauge invariant masses with the $\left\{3,1,-\frac{1}{3}\right\}$ representations present in the $\mathbf{1 5}$ plets, leaving only three $\hat{d}$ 's for the electroweak scale. The sterile neutrino fields, i.e., the $\{1,1,0\}$ representations shown in Eq. (14), can also have mass terms that are invariant under the SM gauge group. If all these masses are large, the only fields that are left over at the electroweak scale are not different from the fields that are obtained in the three generations of $\mathbf{5}^{*}$ and $\mathbf{1 0}$ multiplets of SU(5). Moreover, the model has no explanation for the number of generations. Any number of 15-plets, along with twice the number of $\mathbf{6}^{*}$-plets, would 
be anomaly-free. Hence this model is not interesting for our discussion.

\section{Generalities of SU(N) models with $N>6$}

We now consider models based on the gauge groups $\mathrm{SU}(\mathrm{N})$, with $N>6$. Henceforth we will use completely antisymmetric tensor representations only. Such a representation of rank $n$ will be denoted by $\llbracket n \rrbracket$, as was done in Eq. (16). The fundamental representation will thus be denoted by $\llbracket 1 \rrbracket$ in this notation, whereas its complex conjugate will be $\llbracket N-1 \rrbracket$. We will show that, with antisymmetric representations only, neither the PPF nor the SVS model can be embedded into an $\mathrm{SU}(\mathrm{N})$ grand unified group.

The crucial aspect of both the PPF and the SVS model is that, among the left-chiral fields, the quarks, i.e., color triplets, appear in triplet or antitriplet of SU(3) ${ }_{\mathrm{L}}$, whereas the antiquarks, i.e., color antitriplets, are all singlets of $\mathrm{SU}(3)_{\mathrm{L}}$. In particular, then, there is no multiplet that transforms like $\left[3^{*}, 3, \star\right]$ or $\left[3^{*}, 3^{*}, \star\right]$. On the other hand, among the quark fields, some should be in the $[3,3, \star]$ and some in the $\left[3,3^{*}, \star\right]$ representation, thereby ensuring anomaly cancelation among different generations. These features are not available in the decomposition of any $\llbracket m \rrbracket$ representation of an $\mathrm{SU}(\mathrm{N})$ group, as we show now.

Since $\mathrm{SU}(3)_{\mathrm{c}} \times \mathrm{SU}(3)_{\mathrm{L}} \times \mathrm{U}(1)_{\mathrm{X}}$ is not a maximal subgroup of the groups under consideration, it should be possible to embed the $\mathrm{SU}(3)_{\mathrm{c}} \times \mathrm{SU}(3)_{\mathrm{L}} \times \mathrm{U}(1)_{\mathrm{X}}$ group into the $\mathrm{SU}(\mathrm{N})$ grand unified group in more than one way. First, we assume that the decomposition of the fundamental representation of $\mathrm{SU}(\mathrm{N})$ into $\mathrm{SU}(3)_{\mathrm{c}} \times \mathrm{SU}(3)_{\mathrm{L}} \times \mathrm{U}(1)_{\mathrm{X}}$ is given by

$\llbracket 1 \rrbracket \equiv \mathbf{N} \rightarrow[3,1, \star]+[1,3, \star]+\sum_{k=7}^{N}[1,1, \star]$,

where the $\mathrm{U}(1)_{\mathrm{X}}$ charges have been left unspecified, denoted by the star symbol. There are various ways of assigning the $\mathrm{U}(1)_{\mathrm{X}}$ quantum numbers, and the specifics are irrelevant for our discussion. The representation $\llbracket m \rrbracket$ can contain a $\left[3^{*}, 3, \star\right]$ submultiplet if, among the $m$ tensor indices, two come from the color part and one from the SU(3) $\mathrm{L}$ part, and the remaining $m-3$ indices should belong to the $\mathrm{U}(1)_{\mathrm{X}}$ subgroup. However, the count of $\left[3,3^{*}, \star\right]$ is exactly the same. Thus, we obtain equal numbers of $\left[3^{*}, 3, \star\right]$ and $\left[3,3^{*}, \star\right]$ submultiplets. The only way to get rid of the $\left[3^{*}, 3, \star\right]$ submultiplets is for them to form $\mathrm{SU}(3)_{\mathrm{c}} \times \mathrm{SU}(3)_{\mathrm{L}} \times \mathrm{U}(1)_{\mathrm{X}}$ invariant masses with $\left[3,3^{*}, \star\right]$ submultiplets and become superheavy. But then there is no remaining $\left[3,3^{*}, \star\right]$ submultiplet to contribute to the fermion content of the PPF or the SVS model. Hence the impasse.

The situation is not different if we assume that the decomposition of the fundamental representation follows the rule $\llbracket 1 \rrbracket \equiv \mathbf{N} \rightarrow[3,1, \star]+\left[1,3^{*}, \star\right]+\sum_{k=7}^{N}[1,1, \star]$.

In this case, following the same argument, we would conclude that the number of $[3,3, \star]$ and $\left[3^{*}, 3^{*}, \star\right]$ are equal. Now, the $\left[3^{*}, 3^{*}, \star\right]$ submultiplets can be got rid of by forming superheavy masses with $[3,3, \star]$ submultiplets. But then there will be no $[3,3, \star]$ left at the $\mathrm{SU}(3)_{\mathrm{c}} \times \mathrm{SU}(3)_{\mathrm{L}} \times \mathrm{U}(1)_{\mathrm{X}}$ level, which is unacceptable for both the PPF and the SVS model.

\section{Other embeddings into SU(N)}

At this point, we ignore the intermediate symmetry $\mathrm{SU}(3)_{\mathrm{c}} \times$ $\mathrm{SU}(3)_{\mathrm{L}} \times \mathrm{U}(1)_{\mathrm{X}}$ and try to see whether it is possible to embed the standard model fermions into an SU(N) grand unified group that would provide an explanation for the number of fermion generations. To this end, we first list the decomposition of the lowest rank antisymmetric tensor representations of SU(N) into the SM gauge group. The fundamental representation decomposes as follows:

$\llbracket 1 \rrbracket=\left\{3,1,-\frac{1}{3}\right\}+\left\{1,2, \frac{1}{2}\right\}+(N-5) \cdot\{1,1,0\}$,

where the number within parentheses indicates the number of copies of the SM gauge singlet in the last term. Similarly, we obtain

$$
\begin{aligned}
\llbracket 2 \rrbracket= & \left\{3^{*}, 1,-\frac{2}{3}\right\}+\left\{3,2, \frac{1}{6}\right\}+(N-5) \cdot\left\{3,1,-\frac{1}{3}\right\} \\
& +\{1,1,1\}+(N-5) \cdot\left\{1,2, \frac{1}{2}\right\}+\left(\begin{array}{c}
N-5 \\
2
\end{array}\right) \cdot\{1,1,0\} \\
\llbracket 3 \rrbracket= & \{1,1,-1\}+\left\{3^{*}, 2,-\frac{1}{6}\right\}+(N-5) \cdot\left\{3^{*}, 1,-\frac{2}{3}\right\} \\
& +(N-5) \cdot\left\{3,2, \frac{1}{6}\right\}+\left\{3,1, \frac{2}{3}\right\} \\
& +\left(\begin{array}{c}
N-5 \\
2
\end{array}\right)\left\{3,1,-\frac{1}{3}\right\}+(N-5) \cdot\{1,1,1\} \\
& +\left(\begin{array}{c}
N-5 \\
2
\end{array}\right)\left\{1,2, \frac{1}{2}\right\}+\left(\begin{array}{c}
N-5 \\
3
\end{array}\right)\{1,1,0\} .
\end{aligned}
$$

Suppose now we consider a model with $n_{1}$ copies of $\llbracket 1 \rrbracket$ representation, $n_{2}$ copies of $\llbracket 2 \rrbracket$, and $n_{3}$ copies of $\llbracket 3 \rrbracket$. In counting the 'copies', we will denote a complex conjugate representation by a negative number. We count the number of different SM multiplets in the antisymmetric representations of SU(N) and summarize the result in Table 1. We see that, in order to obtain $n_{g}$ generations of fermions, we need

$n_{2}+(N-6) n_{3}=n_{g}$ 
Table 1 Number of different SM multiplets occurring in completely antisymmetric representations of $\mathrm{SU}(\mathrm{N})$

\begin{tabular}{lccc}
\hline SM multiplet & \multicolumn{2}{l}{ Representation } \\
\cline { 2 - 4 } & $\llbracket 1 \rrbracket$ & $\llbracket 2 \rrbracket$ & $\llbracket 3 \rrbracket$ \\
\hline$\left\{1,2,-\frac{1}{2}\right\}$ & -1 & $-(N-5)$ & $-\left(\begin{array}{c}N-5 \\
2\end{array}\right)$ \\
$\left\{3,2, \frac{1}{6}\right\}$ & 0 & 1 & $N-6$ \\
$\left\{3^{*}, 1,-\frac{2}{3}\right\}$ & 0 & 1 & $N-6$ \\
$\left\{3^{*}, 1, \frac{1}{3}\right\}$ & -1 & $-(N-5)$ & $-\left(\begin{array}{c}N-5 \\
2\end{array}\right)$ \\
$\{1,1,1\}$ & 0 & 1 & $N-6$ \\
\hline
\end{tabular}

in order to ensure the correct number of quark doublets, as well as of $\hat{u}$ and $\hat{\ell}$. In addition, we need

$n_{1}+(N-5) n_{2}+\left(\begin{array}{c}N-5 \\ 2\end{array}\right) n_{3}=-n_{g}$,

so that the correct numbers of lepton doublets and $\hat{d}$ are obtained. Anomaly cancelation between the representations will be ensured if we have

$n_{1}+(N-4) n_{2}+\frac{1}{2}(N-3)(N-6) n_{3}=0$,

making use of the anomaly coefficients given in Eq. (16). However, only two of the three relations in Eq. (21) are independent. We find it simple to work with Eqs. (21a) and (21c). The general solution of these equations is given by

$n_{1}=-(N-4) n_{g}+\frac{1}{2}(N-5)(N-6) n_{3}$,

$n_{2}=n_{g}-(N-6) n_{3}$.

If we take $n_{3}=0$, we obtain $n_{g}=n_{2}$, and hence no explanation of generations. This is what was done for the SU(6) grand unified model discussed in Sect. 3, a model which was found to be uninteresting precisely because it could not predict the number of generations. However, we can consider other kinds of solutions of Eq. (22). For example, if we take $n_{2}=0$, we obtain

$n_{g}=(N-6) n_{3}$.

In this case, for the grand unified group SU(9) we find that the number of generations must be three or its multiple.

It should be noted that it is just as easy to obtain solutions of Eq. (22) with $n_{1}, n_{2}$, and $n_{3}$ all non-zero. One such model with an SU(8) gauge group was the subject matter of Ref. [10], where the authors took $n_{1}=-9, n_{2}=1, n_{3}=1$ and obtained three generations of fermions. From our analysis, it seems that they could have obtained any other number of generations by adjusting the number of copies of various representations. For example, $n_{1}=-13, n_{2}=2, n_{3}=1$ would give four generations. However, the merit of the $n_{2}=0$ solutions is that the number of generations cannot be arbitrary: it must be a multiple of three.

\section{Anatomy of an SU(9) model}

As seen from our earlier analysis, an SU(9) model, in the absence of rank-2 antisymmetric multiplets, automatically gives three fermion generations, provided we take

$n_{3}=1, \quad n_{1}=-9$,

which means that there should be nine copies of the antifundamental representation, and one copy of the rank-3 antisymmetric multiplet.

To see in more detail how different fermion representations of the standard model are obtained from these representations of the grand unified group $\mathrm{SU}(9)$, we first discuss the decomposition of these multiplets under the group $\mathrm{SU}(3)_{\mathrm{c}} \times \mathrm{SU}(3)_{\mathrm{L}} \times \mathrm{U}(1)_{\mathrm{X}}$. The decomposition of the fundamental of SU(9) can be taken to be as given in Eq. (17). We can choose the $U(1)_{X}$ quantum number in a way that it vanishes for all singlets of $\mathrm{SU}(3)_{c} \times \mathrm{SU}(3)_{\mathrm{L}}$. Then, choosing the normalization of the $\mathrm{U}(1)_{\mathrm{X}}$ arbitrarily, we can write

$$
\llbracket \overline{1} \rrbracket \equiv \overline{\mathbf{9}} \rightarrow\left[3^{*}, 1, \frac{1}{3}\right]+\left[1,3^{*},-\frac{1}{3}\right]+3 \cdot[1,1,0] .
$$

This gives, for the rank-3 representation of SU(9), the following decomposition into $\mathrm{SU}(3)_{\mathrm{c}} \times \mathrm{SU}(3)_{\mathrm{L}} \times \mathrm{U}(1)_{\mathrm{X}}$ multiplets:

$$
\begin{aligned}
& \llbracket 3 \rrbracket \equiv \mathbf{8 4}=[1,1,0]+[1,1,1]+[1,1,-1] \\
& +3 \cdot[3,3,0]+\left[3^{*}, 3,-\frac{1}{3}\right]+\left[3,3^{*}, \frac{1}{3}\right] \\
& +3 \cdot\left[3^{*}, 1,-\frac{2}{3}\right]+3 \cdot\left[1,3^{*}, \frac{2}{3}\right]+3 \cdot\left[1,3, \frac{1}{3}\right] \\
& +3 \cdot\left[3,1,-\frac{1}{3}\right] .
\end{aligned}
$$

Looking at these decompositions, we find that the multiplets specified by Eq. (24) contain the following vector-like combinations of $\mathrm{SU}(3)_{\mathrm{c}} \times \mathrm{SU}(3)_{\mathrm{L}} \times \mathrm{U}(1)_{\mathrm{X}}$ submultiplets:

$$
\begin{array}{lll}
28 & \text { copies of } & {[1,1,0],} \\
3 & \text { copies of } & {\left[3^{*}, 1, \frac{1}{3}\right]+\left[3,1,-\frac{1}{3}\right],} \\
3 & \text { copies of } & {\left[1,3^{*},-\frac{1}{3}\right]+\left[1,3, \frac{1}{3}\right],} \\
1 & \text { copy of } & {\left[3^{*}, 3,-\frac{1}{3}\right]+\left[3,3^{*}, \frac{1}{3}\right],} \\
1 & \text { copy of } & {[1,1,1]+[1,1,-1] .}
\end{array}
$$

Such things can have $\mathrm{SU}(3)_{\mathrm{c}} \times \mathrm{SU}(3)_{\mathrm{L}} \times \mathrm{U}(1)_{\mathrm{X}}$ invariant mass terms at the level where the said symmetry is intact, and they do not affect the SM reduction of the model. These singlets and vector-like particles do not contribute to the $\mathrm{SU}(3)_{\mathrm{c}} \times \mathrm{SU}(3)_{\mathrm{L}} \times \mathrm{U}(1)_{\mathrm{X}}$ anomalies.

In addition, we find that the decomposition consists of several chiral multiplets of $\mathrm{SU}(3)_{\mathrm{c}} \times \mathrm{SU}(3)_{\mathrm{L}} \times \mathrm{U}(1)_{\mathrm{X}}$. Under the group $\mathrm{SU}(3)_{\mathrm{L}}$, these multiplets transform either like 
triplets or antitriplets, or like singlets. We want the triplets and antitriplets to contain the doublets of the standard electroweak gauge group. For that, we first have to discuss how the $\mathrm{SM}$ is embedded into $\mathrm{SU}(3)_{\mathrm{c}} \times \mathrm{SU}(3)_{\mathrm{L}} \times \mathrm{U}(1)_{\mathrm{X}}$. Since the members of an $\mathrm{SU}(2)_{\mathrm{L}}$ doublet have the same value of hypercharge $Y$, we need to ensure that the diagonal $Y$ generator has two equal entries in the $3 \times 3$ representation of SU(3) $)_{\mathrm{L}}$. We notice that the PPF model was constructed in such a way that the second and the third elements of a triplet end up with the same value of $Y$ through Eq. (4), whereas in the SVS model, the first two elements are equal. In order to obtain something different, we can now try a solution in which the first and the third elements of $Y$ should have the same diagonal elements. This solution is given by

$I_{3 L}=\frac{1}{2} T_{3}+\frac{\sqrt{ } 3}{2} T_{8}$,

$Y=\frac{1}{2} T_{3}-\frac{1}{2 \sqrt{ } 3} T_{8}+X$.

With these assignments, we now present the chiral multiplets of $\mathrm{SU}(3)_{\mathrm{c}} \times \mathrm{SU}(3)_{\mathrm{L}} \times \mathrm{U}(1)_{\mathrm{X}}$, which are present in the choice of representations in Eq. (24), as well as their SM decompositions:

$3 \cdot[3,3,0] \rightarrow 3 \cdot\left\{3,2, \frac{1}{6}\right\}+3 \cdot\left\{3,1,-\frac{1}{3}\right\}$,

$6 \cdot\left[3^{*}, 1, \frac{1}{3}\right] \rightarrow 6 \cdot\left\{3^{*}, 1, \frac{1}{3}\right\}$,

$6 \cdot\left[1,3^{*},-\frac{1}{3}\right] \rightarrow 6 \cdot\left\{1,2,-\frac{1}{2}\right\}+6 \cdot\{1,1,0\}$,

$3 \cdot\left[3^{*}, 1,-\frac{2}{3}\right] \rightarrow 3 \cdot\left\{3^{*}, 1,-\frac{2}{3}\right\}$,

$3 \cdot\left[1,3^{*}, \frac{2}{3}\right] \rightarrow 3 \cdot\left\{1,2, \frac{1}{2}\right\}+3 \cdot\{1,1,1\}$.

We see that, at the SM level, there are three quark doublets, three multiplets that transform like $\hat{u}_{L}$, and also three that transform like the $\hat{\ell}_{L}$ : precisely the numbers necessary for obtaining the three fermion generations in the SM. We find six SM multiplets that transform like the $\hat{d}_{L}$ in Eq. (29b), but that is not a problem, because three of them can pair up with an equal number of $\left\{3,1,-\frac{1}{3}\right\}$ multiplets as appear in Eq. (29a), and one can obtain a bare mass term that is invariant under the SM gauge group. Similarly, the three $\left\{1,2, \frac{1}{2}\right\}$ multiplets pair up with three of the six $\left\{1,2,-\frac{1}{2}\right\}$ multiplets, leaving three lepton doublets at the SM level. Thus, we are left with exactly the three chiral generations of SM fermions which are necessary for building up the standard model. In addition, there are vector-like combinations of the SM gauge group which come from Eqs. (27) and (29). We write these combinations as follows:

\begin{tabular}{clc}
\hline Notation & $\begin{array}{l}\text { Representations under } \\
\text { the SM gauge group }\end{array}$ & $\begin{array}{c}\text { Number of } \\
\text { copies }\end{array}$ \\
\hline$Q$ & $\left\{3,2, \frac{1}{6}\right\}+\left\{3^{*}, 2,-\frac{1}{6}\right\}$ & 1 \\
$U$ & $\left\{3,1, \frac{2}{3}\right\}+\left\{3^{*}, 1,-\frac{2}{3}\right\}$ & 1 \\
$D$ & $\left\{3,1,-\frac{1}{3}\right\}+\left\{3^{*}, 1,-\frac{1}{6}\right\}$ & 6 \\
$L$ & $\left\{1,2, \frac{1}{2}\right\}+\left\{1,2,-\frac{1}{2}\right\}$ & 6 \\
$E$ & $\{1,1,-1\}+\{1,1,1\}$ & 1 \\
$S$ & $\{1,1,0\}$ & 40 \\
\hline
\end{tabular}

This model is therefore very different from the existing models based on the gauge group $\mathrm{SU}(3)_{\mathrm{c}} \times \mathrm{SU}(3)_{\mathrm{L}} \times \mathrm{U}(1)_{\mathrm{X}}$, the ones outlined in Sect. 2. In the existing models, cancelation of gauge anomalies is obtained by making one fermion generation different from the others. In the present model, this is not the case. At the level of the $\mathrm{SU}(3)_{\mathrm{c}} \times \mathrm{SU}(3)_{\mathrm{L}} \times \mathrm{U}(1)_{\mathrm{X}}$ group, all fermion generations have the same transformation properties. However, there are extra fermions that help cancel the anomalies, fermions which become vector-like at the SM level. Thus, even apart from the grand unification prospects via $\mathrm{SU}(9)$, the multiplets presented in Eq. (29) can be taken to represent a new $\mathrm{SU}(3)_{\mathrm{c}} \times \mathrm{SU}(3)_{\mathrm{L}} \times \mathrm{U}(1)_{\mathrm{X}}$ model, which can be studied in its own right. However, if we consider it purely as a $\mathrm{SU}(3)_{\mathrm{c}} \times \mathrm{SU}(3)_{\mathrm{L}} \times \mathrm{U}(1)_{\mathrm{X}}$ model, there is no explanation of the number of generations. One can obtain any number of generations by changing the number of copies of each multiplet, keeping the ratios intact, and obtain any number of generation one wants. Thus, it is fair to say that the explanation of the number of generations comes directly from the grand unification group $\mathrm{SU}(9)$, and not from its subgroup $\mathrm{SU}(3)_{\mathrm{c}} \times \mathrm{SU}(3)_{\mathrm{L}} \times \mathrm{U}(1)_{\mathrm{X}}$.

\section{Gauge coupling unification and the intermediate scales}

We now address the unification picture of the SU(9) model defined by the condition given in Eq. (24). For the sake of simplicity, we shall assume in our analysis that the SU(9) GUT model is spontaneously broken, through the scheme given by Eq. (19), directly to the SM gauge group at a unique unification scale $\Lambda$. Thus, the unification condition for the SM gauge couplings $\alpha_{1,2,3}$ at the scale $\Lambda$ reads

$\alpha_{U}=k_{1} \alpha_{1}(\Lambda)=k_{2} \alpha_{2}(\Lambda)=k_{3} \alpha_{3}(\Lambda)$.

The normalization constants $k_{i}$ are defined by

$k_{i} \equiv \frac{\operatorname{tr} T_{i}^{2}}{\operatorname{tr} T^{2}}$,

where $T$ and $T_{i}$ are the same unbroken generator properly normalized to the GUT group and its subgroup $G_{i}$, respectively, for a given representation. Taking into account the 
decomposition of the fundamental representation given in Eq. (19) and the GUT normalization in Eq. (3), one easily derives that the normalization constants $k_{i}$ take the canonical values

$k_{1}=\frac{5}{3}, \quad k_{2}=k_{3}=1$,

like in the SU(5) GUT models. The relation given in Eq. (31) is only valid at the unification scale and one has to relate these gauge couplings with measurable quantities at the electroweak scale.

The evolution of the gauge couplings in the one-loop approximation is ruled by the solutions of the Renormalization Group Equations (RGEs), which depend on the masses of the particles in the model. We take the SM particles and $n_{\mathrm{H}}$ Higgs doublets at the electroweak scale. In addition, the vector-like combinations given in Eq. (30) can also have masses between $M_{Z}$ and the unification scale $M_{U}$. We will assume, for the sake of simplicity, that all extra vector-like fermions with the same quantum number share their mass scales, and we denote these scales collectively as $M_{I}$, where $I$ can take the 'values' $Q, U, D, L, E$ as shown in Eq. (30). The solutions of the RGEs can then be written as

$\alpha_{1}^{-1}(\mu)=\alpha_{1}^{-1}\left(M_{Z}\right)-\frac{b_{1}}{2 \pi} \ln \left(\frac{\mu}{M_{Z}}\right)-\sum_{I} \frac{b_{1}^{I}}{2 \pi} \ln \left(\frac{\mu}{M_{I}}\right)$,

$\alpha_{2}^{-1}(\mu)=\alpha_{2}^{-1}\left(M_{Z}\right)-\frac{b_{2}}{2 \pi} \ln \left(\frac{\mu}{M_{Z}}\right)-\sum_{I} \frac{b_{2}^{I}}{2 \pi} \ln \left(\frac{\mu}{M_{I}}\right)$,

$\alpha_{3}^{-1}(\mu)=\alpha_{3}^{-1}\left(M_{Z}\right)-\frac{b_{3}}{2 \pi} \ln \left(\frac{\mu}{M_{Z}}\right)-\sum_{I} \frac{b_{3}^{I}}{2 \pi} \ln \left(\frac{\mu}{M_{I}}\right)$,

where the $b_{i}$ are the one-loop beta coefficients that take into account the quantum numbers of the SM fermions, the gauge bosons, and $n_{\mathrm{H}}$ Higgs doublets:

$b_{1}=\frac{20}{9} n_{g}+\frac{n_{\mathrm{H}}}{6}, \quad b_{2}=\frac{4}{3} n_{g}+\frac{n_{\mathrm{H}}}{6}-\frac{22}{3}$,

$b_{3}=\frac{4}{3} n_{g}-11$.

The $b_{i}^{I}$ are the one-loop beta coefficients for the intermediate extra vector-like fermions. The values of these $b_{i}^{I}$ are given in Table 2.

Table 2 Beta coefficients for the extra vector-like fermions

\begin{tabular}{llllll}
\hline & $Q$ & $U$ & $D$ & $L$ & $E$ \\
\hline$b_{1}$ & $2 / 9$ & $16 / 9$ & $4 / 9$ & $2 / 3$ & $4 / 3$ \\
$b_{2}$ & 2 & 0 & 0 & $2 / 3$ & 0 \\
$b_{3}$ & $4 / 3$ & $2 / 3$ & $2 / 3$ & 0 & 0 \\
\hline
\end{tabular}

In order to get some insight into the unification in the one-loop approximation, i.e. to understand the intermediate scales which lead to successful unification, let us define the effective beta coefficients $B_{i}[11,12]$,

$B_{i} \equiv \frac{1}{k_{i}}\left(b_{i}+\sum_{I} b_{i}^{I} r_{I}\right)$,

where the ratio $r_{I}$ is given by

$r_{I}=\frac{\ln \left(\Lambda / M_{I}\right)}{\ln \left(\Lambda / M_{Z}\right)}$.

It is also convenient to introduce the differences $B_{i j} \equiv B_{i}-$ $B_{j}$, such that

$B_{i j}=B_{i j}^{\mathrm{SM}}+\sum_{I} \Delta_{i j}^{I} r_{I}$,

where $B_{i j}^{\mathrm{SM}}$ corresponds to the SM particle contribution and $\Delta_{i j}^{I}=\frac{b_{i}^{I}}{k_{i}}-\frac{b_{j}^{I}}{k_{j}}$.

We then find that

$$
\begin{aligned}
& B \equiv \frac{B_{23}}{B_{12}}=\frac{\sin ^{2} \theta_{W}-\frac{k_{2}}{k_{3}} \frac{\alpha}{\alpha_{s}}}{\frac{k_{2}}{k_{1}}-\left(1+\frac{k_{2}}{k_{1}}\right) \sin ^{2} \theta_{W}}, \\
& B_{12} \ln \left(\frac{\Lambda}{M_{Z}}\right)=\frac{2 \pi}{\alpha}\left[\frac{1}{k_{1}}-\left(\frac{1}{k_{1}}+\frac{1}{k_{2}}\right) \sin ^{2} \theta_{W}\right] .
\end{aligned}
$$

Notice that the right-hand sides of Eq. (40) depend only on low-energy electroweak data and the group factors $k_{i}$. Adopting the following experimental values at $M_{Z}$ [13]:

$\alpha^{-1}=127.916 \pm 0.015$,

$\sin ^{2} \theta_{W}=0.23116 \pm 0.00012$,

$\alpha_{s}=0.1184 \pm 0.0007$,

the above relations give

$B=0.718 \pm 0.003$,

$B_{12} \ln \left(\frac{\Lambda}{M_{Z}}\right)=185.0 \pm 0.2$,

in the canonical GUT models with $k_{i}=(5 / 3,1,1)$. The coefficients $B_{i j}$, which appear in the left-hand sides of Eq. (40), strongly depend on the particle content of the theory. For instance, considering the SM-like particles, with $n_{g}$ generations, together with $n_{\mathrm{H}}$ light Higgs doublets, one has for the coefficients $B_{12}$ and $B_{23}$ :

$B_{12}=\frac{22}{3}-\frac{n_{\mathrm{H}}}{15}, \quad B_{23}=\frac{11}{3}+\frac{n_{\mathrm{H}}}{6}$.

In the case of SM, i.e. $n_{g}=3$ and $n_{\mathrm{H}}=1$ one has

$B=115 / 218 \approx 0.53$, 
Table 3 Beta coefficients for the extra vector-like fermions

\begin{tabular}{lccccl}
\hline & $Q$ & $U$ & $D$ & $L$ & $E$ \\
\hline$B_{12}$ & $-28 / 15$ & $16 / 15$ & $4 / 15$ & $-4 / 15$ & $4 / 5$ \\
$B_{23}$ & $2 / 3$ & $-2 / 3$ & $-2 / 3$ & $2 / 3$ & 0 \\
\hline
\end{tabular}

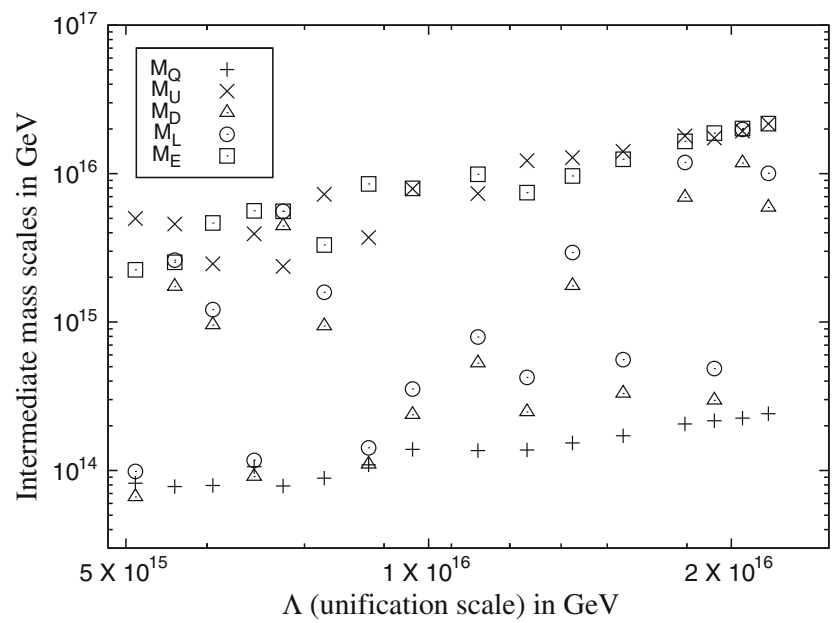

Fig. 1 The intermediate scales of the extra vector-like fermions in function of the unification scale $\Lambda$

which is not compatible with the calculated value in Eq. (42) and, clearly, the B-test fails badly in the SM. In Table 3, we have summarized the contributions of the vector-like fermions to $B_{12}$ and $B_{23}$. Using these values, we have taken random values of the intermediate scales of the extra vectorlike fermions and found combinations of these intermediate scales which are compatible with successful unification. In our calculations we have assumed only one Higgs doublet, i.e. $n_{\mathrm{H}}=1$, and we have taken a rough lower bound on the unification scale, $M_{U}>6 \times 10^{15} \mathrm{GeV}$, coming from the unobservability of proton decay [13] into $e^{+} \pi^{0}$. A set of a million of such random combinations were taken. A few of the allowed ones are presented in Fig. 1. From the entire set of combinations used in our calculations, we find that the allowed range for the unification scale obtained is

$6 \times 10^{15} \mathrm{GeV} \leq \Lambda \leq 2.2 \times 10^{16} \mathrm{GeV}$,

which is also roughly what the limited data of Fig. 1 indicates. For the scales of the vector-like extra fermions we have

$5.5 \times 10^{13} \mathrm{GeV} \leq M_{Q} \leq 2.4 \times 10^{14} \mathrm{GeV}$,

$1.2 \times 10^{15} \mathrm{GeV} \leq M_{U} \leq 2.2 \times 10^{16} \mathrm{GeV}$,

$6.6 \times 10^{13} \mathrm{GeV} \leq M_{D} \leq 1.2 \times 10^{16} \mathrm{GeV}$,

$7.4 \times 10^{13} \mathrm{GeV} \leq M_{L} \leq 2.0 \times 10^{16} \mathrm{GeV}$,

$1.7 \times 10^{15} \mathrm{GeV} \leq M_{E} \leq 2.1 \times 10^{16} \mathrm{GeV}$.

We see that the range of mass scales of the vector-like fermions are high, but they are indeed necessary for driving the evolution of the gauge couplings to perfect unification. If one varies the number of Higgs doublets, $n_{\mathrm{H}}=2,3$, the ranges of the intermediate scales above do not change significantly.

\section{Conclusions}

To summarize, we have succeeded in building a grand unified model based on the group SU(9). The model uses fermions in antisymmetric representations only, and the consistency of the model demands that the number of fermion generations is three. As mentioned in the introduction, earlier models based on the gauge group $\mathrm{SU}(3)_{\mathrm{c}} \times \mathrm{SU}(3)_{\mathrm{L}} \times \mathrm{U}(1)_{\mathrm{X}}$ and $\mathrm{SO}(18)$ also had this property of predicting the number of generations. It is interesting to note that our group $\mathrm{SU}(9)$ contains $\mathrm{SU}(3)_{\mathrm{c}} \times \mathrm{SU}(3)_{\mathrm{L}} \times \mathrm{U}(1)_{\mathrm{X}}$, and that it is contained in $\mathrm{SO}(18)$. However, in our model, the transformation properties of the known fermions in the $\mathrm{SU}(3)_{\mathrm{c}} \times \mathrm{SU}(3)_{\mathrm{L}} \times \mathrm{U}(1)_{\mathrm{X}}$ subgroup of $\mathrm{SU}(9)$ are not the same as those used in earlier models based on the $\mathrm{SU}(3)_{\mathrm{c}} \times \mathrm{SU}(3)_{\mathrm{L}} \times \mathrm{U}(1)_{\mathrm{X}}$ group. This is different where the known fermions of all generations transform in the same way under $\mathrm{SU}(3)_{\mathrm{c}} \times \mathrm{SU}(3)_{\mathrm{L}} \times \mathrm{U}(1)_{\mathrm{X}}$. On the other hand, a comparison with the $\mathrm{SO}(18)$ models also reveals an interesting connection. In $\mathrm{SO}(18)$ models, the fermion generations are contained in the spinor representation. The spinor representation of $\mathrm{SO}(18)$, which is 256-dimensional, decomposes under the $\mathrm{SU}(9)$ subgroup as follows:

$\mathbf{2 5 6}=\llbracket 1 \rrbracket+\llbracket 3 \rrbracket+\llbracket 5 \rrbracket+\llbracket 7 \rrbracket+\llbracket 9 \rrbracket$.

Our SU(9) model uses only the first two kinds of these submultiplets to predict the number of fermion generations.

We have also shown that our model may be consistent with unification requirements. In this part of the analysis, we have assumed a direct breaking of SU(9) into the SM gauge group. A more detailed analysis, including possibilities of intermediate symmetry breaking scales, will be taken up in a future work.

Acknowledgments The work of D.E.C. was supported by Associação do Instituto Superior Técnico para a Investigação e Desenvolvimento (IST-ID) and also by FCT through the projects PEst-OE/FIS/UI0777/ 2011 (CFTP), CERN/FP/123580/2011 and PTDC/FIS-NUC/0548/2012.

Open Access This article is distributed under the terms of the Creative Commons Attribution License which permits any use, distribution, and reproduction in any medium, provided the original author(s) and the source are credited.

Funded by $\mathrm{SCOAP}^{3}$ / License Version CC BY 4.0.

\section{References}

1. F. Wilczek, A. Zee, Families from spinors. Phys. Rev. D 25, 553 (1982) 
2. D. Chang, T. Hubsch, R.N. Mohapatra, Grand unification of three light generations. Phys. Rev. Lett. 55, 673 (1985)

3. T. Hubsch, P.B. Pal, Economical unification of three families in SO(18). Phys. Rev. D 34, 1606 (1986)

4. M. Singer, J.W.F. Valle, J. Schechter, Canonical neutral current predictions from the weak electromagnetic gauge group $\mathrm{SU}(3) \times$ U(1). Phys. Rev. D 22, 738 (1980)

5. F. Pisano, V. Pleitez, An SU(3) $\times$ U(1) model for electroweak interactions. Phys. Rev. D 46, 410-417 (1992)

6. P.H. Frampton, Chiral dilepton model and the flavor question. Phys. Rev. Lett. 69, 2889-2891 (1992)

7. J.W.F. Valle, M. Singer, Lepton number violation with quasi Dirac neutrinos. Phys. Rev. D 28, 540 (1983)
8. R. Foot, H.N. Long, T.A. Tran, $\mathrm{SU}(3)_{\mathrm{L}} \times \mathrm{U}(1)_{\mathrm{N}}$ and $\mathrm{SU}(4)_{\mathrm{L}} \times$ $\mathrm{U}(1)_{\mathrm{N}}$ gauge models with right-handed neutrinos. Phys. Rev. D 50, 34-38 (1994)

9. J. Banks, H. Georgi, Comment on gauge theories without anomalies. Phys. Rev. D 14, 1159-1160 (1976)

10. R. Martinez, F. Ochoa, P. Fonseca, A 3-3-1 Model with SU(8) unification (2011). arXiv:1105.4623 [hep-ph]

11. A. Giveon, L.J. Hall, U. Sarid, SU(5) unification revisited. Phys. Lett. B 271, 138-144 (1991)

12. D. Emmanuel-Costa, E.T. Franco, R.G. Felipe, $\mathrm{SU}(5) \times \mathrm{SU}(5)$ unification revisited. JHEP 1108, 017 (2011)

13. J. Beringer et al., Review of particle physics (RPP). Phys. Rev. D 86, 010001 (2012) 